
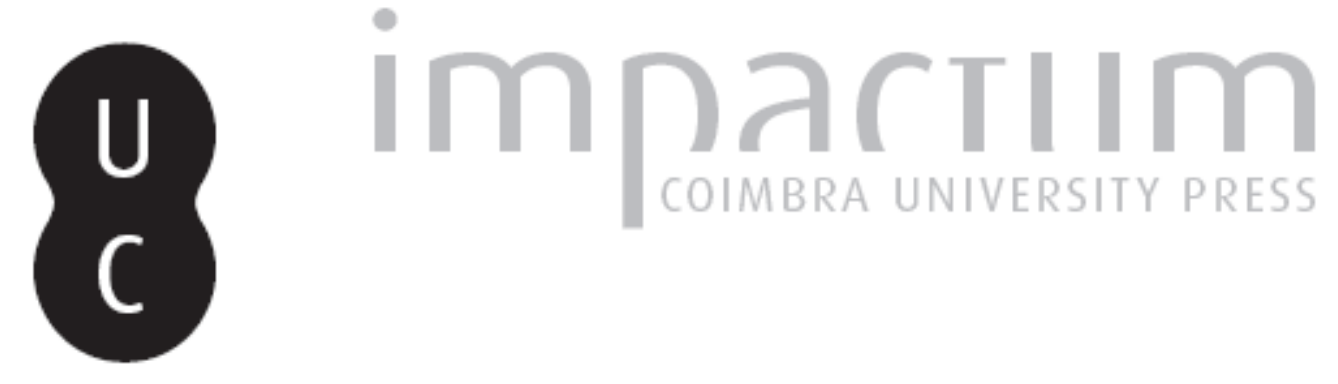

\title{
Une histoire de la politique régionale européenne
}

\section{Autor(es): $\quad$ Alliès, Paul}

Publicado por: Centro de Informação Europe Direct de Aveiro; Centro de Estudos \section{Interdisciplinares do Século XX}

URL

persistente:

DOI:

URI:http://hdl.handle.net/10316.2/37106

Accessed : $\quad$ 26-Apr-2023 15:39:39

DOI:http://dx.doi.org/10.14195/1647-6336_12_1

A navegação consulta e descarregamento dos títulos inseridos nas Bibliotecas Digitais UC Digitalis, UC Pombalina e UC Impactum, pressupõem a aceitação plena e sem reservas dos Termos e Condições de Uso destas Bibliotecas Digitais, disponíveis em https://digitalis.uc.pt/pt-pt/termos.

Conforme exposto nos referidos Termos e Condições de Uso, o descarregamento de títulos de acesso restrito requer uma licença válida de autorização devendo o utilizador aceder ao(s) documento(s) a partir de um endereço de IP da instituição detentora da supramencionada licença.

Ao utilizador é apenas permitido o descarregamento para uso pessoal, pelo que o emprego do(s) título(s) descarregado(s) para outro fim, designadamente comercial, carece de autorização do respetivo autor ou editor da obra.

Na medida em que todas as obras da UC Digitalis se encontram protegidas pelo Código do Direito de Autor e Direitos Conexos e demais legislação aplicável, toda a cópia, parcial ou total, deste documento, nos casos em que é legalmente admitida, deverá conter ou fazer-se acompanhar por este aviso.

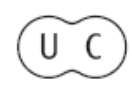


DEBATER

A EUROPA

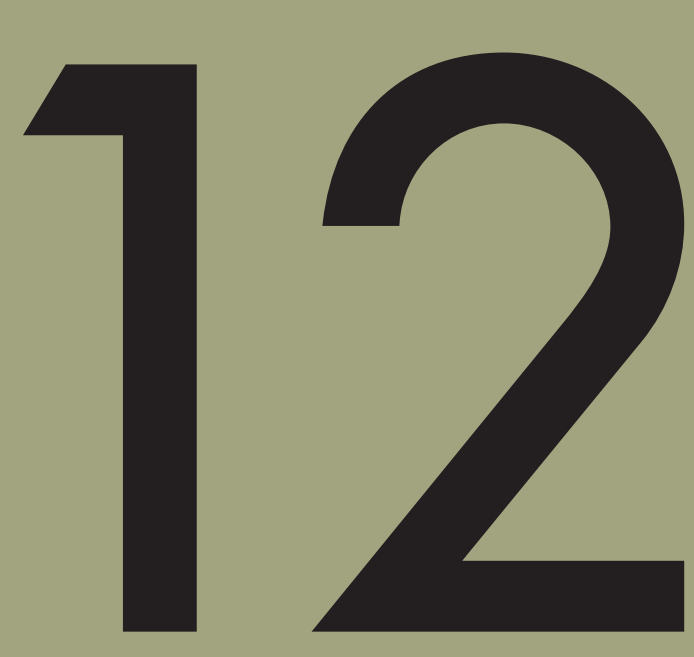

jan-jun 2015

POLITICAS REGIONAIS DA UE

EU REGIONAL POLICIES 


\title{
Une histoire de la politique régionale européenne
}

\author{
Paul Alliès \\ Professeur de droit \\ Université de Montpellier 1, France \\ E-mail : paul.allies@univ-montp1.fr
}

\section{Résumé}

Le régionalisme a toujours été dans l'Europe post-Westphalienne une question incertaine, administrée de manière contradictoire par les Etats-nations ou les Empires. Son traitement dans le cadre des institutions européennes de l'après-guerre a marqué un tournant essentiel. Alors qu'il n'avait fait l'objet d'aucune définition dans les Traités, la Communauté-Union Européenne a réussi à en faire une politique essentielle à sa propre construction. La situation a complètement changé après l'adoption par les Etats membres de la stratégie de Lisbonne en 2000. On tentera ici de proposer une lecture de ce cycle régionaliste, riche en pratiques et en résultats pour un avenir qui n'est pas tracé.

Mots-clés: régionalisme ; Union Européenne ; stratégie de Lisbonne ; politique régionale européenne

\section{1 - La définition d'un cadre d'action régional sui generis.}

On partira d'un fort paradoxe : les traités fondateurs de la Communauté Européenne ne prévoyaient en rien une quelconque politique régionale commune. Il faut s'interroger sur les raisons de cette absence. Mais la dynamique institutionnelle et politique de la construction européenne a fait émerger le régionalisme au premier rang des interventions de l'Union Européenne (UE). Au point que l'on puisse affirmer que c'est par la politique régionale que l'Europe s'est imposée aux Etats. 


\subsection{La justification d'une politique régionale :}

L'Europe n'a été jusqu'ici et pour l'essentiel qu'une coordination structurée par des traités des gouvernements d'Etats-nations ${ }^{1}$. L'idée qu'elle puisse avoir une politique régionale n'allait nullement de soi puisque ce secteur des politiques publiques relevait de l'apanage de chaque Etat-membre. Elle ne s'est imposée que tardivement et presque par défaut. D'abord en reconnaissant la Région comme catégorie politique légitime. Ensuite en l'adoptant comme niveau pertinent de politiques publiques.

\subsubsection{La Région comme catégorie problématique :}

Le terme même de « régionalisme » a toujours fait débat ${ }^{2}$ : à la fin du XIX ${ }^{\circ}$ siècle, il fut promu dans toute l'Europe en idéologie de résistance à l'industrialisation ou d'assimilation de la construction nationale des sociétés. C'est dans les années 1880 qu'éclot un mouvement régionaliste à la fois sur le plan culturel et plus spécialement littéraire, comme sur le plan politique. On le rencontre aussi bien dans les pays de langue allemande qu'en Scandinavie ou en Europe du Sud. Il a en commun une défense des «valeurs » de la société rurale et d'un nationalisme fondé sur la défense de la «petite patrie » comme ciment de loyauté à la grande. Ce mouvement est donc typique d'un double processus historiquement daté : l'attachement à un mode de vie traditionnel contre les progrès de l'urbanisation et de l'industrialisation; la valorisation des territoires d'appartenance conçus comme le socle du pacte social de l'Etat-nation. Dans les deux cas, les modes de vie des habitants des campagnes apparaissent comme une matérialisation du peuple telle que le requiert la nouvelle «personnalité nationale». La construction symbolique de celle-ci se fait, non plus sur l'obéissance au souverain mais sur le partage de traditions populaires. Ce sera le moment de l'essor de la géographie humaine, de l'anthropologie culturaliste, du roman provincialiste, de l'architecture régionaliste et du tourisme folklorique. Cet intérêt présent dans toute l'Europe post-romantique a des traductions politiques dans deux directions différentes: dans la revendication de réformes administratives et institutionnelles soutenue par les élites favorables à la décentralisation-régionalisation de l'Etat d'une part; dans la rhétorique réactionnaire $\mathrm{du}$ « retour à la terre » réclamé par la droite nationaliste d'autre part.

\footnotetext{
${ }^{1}$ QUERMONNE, Jean-Louis - L'Union européenne dans le temps long. Préface de Jacques Delors. Paris : Les presses de Sciences Po, 2008. $231 \mathrm{p}$.

2 ALLIES, Paul - Nationalités, nationalitaire, régionalisme - In ARTOUS Antoine, EPSZTAJN Didier, SIBLBERSTEIN Patrick (dir.) La France des années 1968. Paris : Ed. Syllepses., 2008. pp. 534-549
} 
Aussi au lendemain de la $2^{\circ}$ guerre mondiale, le régionalisme prit un nouveau cours. Il va apparaître comme la voie moyenne de mise en cause de l'Etat-nation devenu la forme problématique du nationalisme responsable, dans sa version hitlérienne mais aussi mussolinienne, du conflit. Tout se passait comme si l'Etat-nation était resté depuis le XIX ${ }^{\circ}$ siècle, le ferment de progrès politiques et sociaux tels que la Société Des Nations puis et maintenant les Nations Unies les prenaient en compte. Or il était désormais dénoncé comme étant la source d'oppressions et de négations en particulier dans le domaine des cultures et des communautés linguistiques que le Conseil de l'Europe allait prendre en charge de mieux en mieux.

\subsubsection{La Région comme catégorie légitime :}

C'est ainsi que les pères fondateurs de l'Europe (aussi bien Monnet que De Gasperi, Adenauer ou Schuman) imaginèrent un dépassement de l'Etat-nation dans un fédéralisme «sui generis » qui prit souvent le nom d' «Europe des Régions » ${ }^{3}$. Les différentes versions du régionalisme vont être reçues dans le double mouvement du développement de la politique régionale de l'UE et de ses élargissements successifs. Par exemple, l'adhésion britannique jouera un rôle décisif dans la naissance du FEDER en 1985 puis les adhésions méditerranéennes dans la réforme de $1988^{4}$. Le contenu de la politique régionale communautaire va se spécialiser toujours plus sous les vocables de politique «structurelle » ou de «cohésion». Une méthode de répartition des fonds par indicateurs régionaux, en partenariat avec les collectivités locales, ainsi que des conditions d'éligibilité aux programmes communautaires va concrétiser l'Europe des régions de façon très fonctionnelle. Elle accompagne le «New Public Management » qui vise à restreindre les interventions de l'Etat dans le domaine social et économique. Les Régions deviennent le cadre potentiel de mobilisation de la «société civile» et de définition de nouveaux rapports entre privé et public $^{5}$. Elles servent de base à la définition d'un nouveau système de «gouvernance à niveaux multiples » qui transforme profondément les rapports entre régions et instances nationales et européennes. La formalisation institutionnelle de cela est, à partir des années Quatre-vingt dans la création d'instances comme la Conférence des Régions Maritimes et

\footnotetext{
${ }^{3}$ SCHWOK, René - Théories de l'intégration européenne. Paris : Montchrestien, 2005. 154p.

${ }^{4}$ VANHOVE Norbert ; H. KLAASSEN Léo - Régional Policy, a European approach. Avebury: Aldershot, $1987.589 \mathrm{p}$.

${ }^{5}$ FALUDI Andreas, WATERHOUT Bas - The making of the European Spatial Development Perspective, No Masterplan. London and New York : Routledge. 2002, 194 p.
} 
Périphériques (CRMP) ou l'Assemblée des Régions Européennes (ARE) et finalement du Comité des Régions par le traité de Maastricht.

C'est donc une des réussites de l'UE que d'avoir dénationalisé et capté la vieille revendication égalitariste des territoires en retard de développement industriel dans la période de longue durée (1850-1950), en objectif de cohésion économique et sociale dans les années 1950-2000 (on va voir comment la période 1958-1975, année de la création du premier Fonds régional donne bien la mesure de cette transformation). Ce qui s'est joué là, c'est la reconversion historique du régionalisme. En 1968 les revendications des périphéries étatiques accédaient au centre de la protestation quels que soient les régimes ou les formations nationales. Elles n'allaient plus la quitter au cours de la décennie qui allait suivre. Cette idée fut donc tributaire de deux transformations historiques de l'Etat :

1) L'autonomie et la souveraineté nationale sont devenues de plus en plus fictives tant dans l'ordre interne qu'international. L'intégration enclenchée par les traités est apparue comme une méthode efficace pour faire face à la succession des crises économiques survenants après la période de reconstruction. Le chômage, l'inflation, la stagnation rythmaient la remise en cause du modèle keynésien qui avait triomphé un peu partout à l'issue du conflit mondial. La concurrence du Japon et des Etats-Unis encourageait la mutualisation du marché européen sur la base de la libre concurrence et donc de l'abaissement des frontières nationales ${ }^{6}$. Le traité de Maastricht puis celui d'Amsterdam ont consacré ce processus au terme duquel virent le jour un marché, une monnaie et une banque unique. Les gouvernements nationaux ont ainsi abandonné des compétences régaliennes essentielles.

2) Les autorités subnationales (régionales) se virent promues dans un statut et une fonction qui dépassaient largement le cadre culturel ou administratif qui les identifiaient jusque-là. Jusque dans les années Quatre-vingt elles étaient restées sous le contrôle quasi absolu du gouvernement central. Même dans les systèmes fédéraux comme en Allemagne, la tendance était à la centralisation des finances publiques. Or la scène européenne va voir les Régions (mais aussi les villes) devenir des entités indispensables aux réformes néo-libérales de l'Etat. A partir de 1985 la création des «Fonds structurels » a engendré la promotion de nouvelles notions comme celles de

\footnotetext{
${ }^{6}$ LUNDESTAD, Geir - Europamerique. Etats-Unis et Europe de l'Ouest de 1945 à nos jours. Paris : Eyrolles, 2013. $456 \mathrm{p}$.
} 
«subsidiarité » et de «partenariat ${ }^{7}$. Les Länder allemands ont initié le mouvement en faveur d'une Europe des Régions qui a trouvé un écho important de la Catalogne à l'Emilie-Romagne. Là s'est dessiné un nouveau modèle de développement régional (baptisé «the learning region »), à l'époque présenté comme alternatif aux modèles étatiques: le régionalisme était l'autre nom de réseaux denses d'entrepreneurs, de systèmes de formation, de cultures de coopération entre acteurs sociaux et économiques, d'ouverture à l'innovation et à l'international. L'essor de ce régionalisme apparaissait comme une condition de l'intégration de sociétés spécifiques au marché mondial indépendamment des Etats-nations.

C'est ainsi que l'Union Européenne a pu institutionnaliser un régionalisme fonctionnel dans tous ses Etat-membres y compris les plus centralisés comme la Grèce, l'Irlande ou le Portugal.

Le processus s'est concrètement engagé à partir de la prise en compte des disparités régionales et des retards de développement entre régions industrielles et régions restées préindustrielles (et d'abord celles du sud de l'Europe, France incluse). Mais il y eut aussi le cas des Régions marquées par le déclin industriel (à partir des années Trente en Angleterre puis dans toute l'Europe continentale à partir des années Soixante dans le charbon et la sidérurgie). Ces Régions apparaissaient comme des «poches de pauvreté » (cf. «Le Christ s'est arrêté à Eboli » de Carlo Levi en 1945 sur Matera dans le Basilicate au sud de l'Italie) : les leaders democrates-chretiens (De Gasperi) tiennent un discours selon lequel les démocraties ayant gagné la guerre ne peuvent accepter de telles injustices dans la nouvelle Europe.

Chacun des Etats va alors construire des instruments de correction des mécanismes du marché et de son développement inégal. L'Italie avec la Cassa per il Mezzogiorno (crée en 1950, supprimée en 1986) ; le Royaume-Uni avec les Distribution of Industriy Act (de 1945 à 1980) pour les cinq régions en déclin; en France avec la DATAR (Direction de l'Aménagement du Territoire et de la Politique Régionale) de 1963 - en réalité 1956 sous un autre nom- à la fin des années Quatre-vingt; en Allemagne où les Länder ont reçu une compétence forte dans l'aménagement du territoire. Durant cette période, les Etats-nations ont renforcé leur légitimité avec ces politiques régionales complétant leurs politiques keynésiennes : les politiques régionales se présentaient comme un aspect des politiques

\footnotetext{
${ }^{7}$ CHARLES LE-BIHAN, Danielle - Les instruments juridiques de l'Aménagement du territoire de l'Union Européenne. Rennes : Presses Universitaires, 2004. 260 p.
} 
d'industrialisation et des stratégies de développement. Les politiques régionales vont d'ailleurs être réputées protectionnistes d'un point de vue communautaire. Seule la CECA (crée par l'art. 56 du Traité de Paris du 18 avril 1951) a ouvert une porte en ciblant les zones affectées par la crise du charbon et de l'acier. Le Traité de Rome (25 mars 1957) prévoit (art. 130) la création d'une Banque Européenne d'Investissement qui pourra prendre en considération les inégalités régionales dans l'agriculture. C'est la très mince ouverture sur une dimension régionale. Et c'est seulement à partir de la mise en place de la politique agricole commune en 1963 qu'on prend conscience que le «marché commun » peut aggraver les disparités régionales au détriment des régions agricoles en particulier. C'est de là qu'est venue la première mise au point d'une politique régionale communautaire.

\subsection{La Région comme niveau de politique publique}

Dix ans après le Traité de Rome la politique régionale n'avait toujours pas de base juridique et rencontrait politiquement l'opposition diamétralement opposée des libéraux et des souverainistes $^{8}$. La Commission s'est alors engagée dans une démarche où elle a présenté la politique régionale comme un complément ou un contrepoids à la politique de concurrence : les régions étaient présentées comme un moyen de lever les obstacles à la libre circulation des capitaux, des marchandises et des Hommes. La présidence Delors (6 janvier 1985 - 5 janvier 1995) sera celle durant laquelle intégration européenne et politiques territoriales seront étroitement liées et feront l'objet d'interventions proprement européennes.

\subsubsection{Le Fonds Européen de Développement Régional (FEDER)}

La création du FEDER s'effectue entre la conférence des chefs d'Etats et de Gouvernements d'octobre 1972 qui établit la politique régionale comme «un élément nécessaire à côté de l'union économique et monétaire pour renforcer la Communauté » et le premier règlement le définissant en mars $1975^{9}$. Cela a été possible car la perspective de l'adhésion du Royaume-Uni, de l'Irlande, du Danemark et de la Norvège exigeait de trouver des compensations budgétaires pour ces pays qui n'ont pas d'agriculture importante pour leur PIB. Les Etats ont donc affecté un budget de 1300 Millions d'équivalents Euros pour trois ans

\footnotetext{
${ }^{8}$ DREVET, Jean-François - Histoire de la politique régionale de l'Union européenne. Paris : Belin, 2008. 284 p.

${ }^{9}$ Idem, ibid. p. 51 et s.
} 
avec un système de répartition par quotas. Cela veut dire que le budget est réparti par négociation entre les Gouvernements et non pas suivant une appréciation objective des problèmes régionaux. La Commission doit donc subventionner des «projets d'investissements productifs » qui lui sont en fait proposés par les gouvernements (auxquels les collectivités locales ne sont même pas associées). Mais pour faire cela, elle doit mettre au point un instrument administratif pour sélectionner, encadrer et évaluer chaque projet retenu. Cela ouvre la voie à une construction politique. (la méthode Monnet connue sous le vocable de «spill over »).

Le budget du FEDER va en effet connaître une croissance rapide entre 1975 et 1984. Même s'il ne pèse que $7 \%$ du budget communautaire majoritairement consommé par la Politique Agricole Commune, il aura quadruplé dans ces dix ans. La Commission fera admettre au Conseil une direction spécifique (la DG XVI qui s'appellera ensuite REGIO); elle est créée en 1968 pour gérer ces fonds. Elle gagne un poids très important au fur et à mesure qu'on lui soumet les projets (26.000 entre 1975 et 1984). Elle va faire valoir une capacité d'expertiser notamment pour spécifier les opérations improductives ou même non réalisées. Elle démontre ainsi l'absurdité d'un système (de quotas) qui vise à abonder la trésorerie des Etats plus que de produire de la valeur ajoutée régionale. Elle alimente ainsi l'audit de la Cour des Comptes qui dénonce en 1984 le fait que 42\% des projets sélectionnés (3000) depuis 1975 n'ont pu être suivi de paiements. Pour autant les opérations menées à bien (1700) ont généré 640.000 emplois et aidé au développement régional.

C'est donc logiquement que le Conseil autorise la Commission en février 1979 à sélectionner des projets hors quota dans la limite de 5\% de la dotation FEDER. C'est peu : il faudra attendre le sommet de Berlin en 1999 pour que la Commission soit autorisée à utiliser sa propre méthode objective de répartition des fonds et de sélection des dossiers. Mais c'est parce qu'entre-temps la Commission a su développer les synergies entre les trois Fonds dits structurels (FEDER, FEOGA, FSE). La définition de nouvelles unités-statistiques territoriales (NUTS) réalisées par Eurostat, permet de définir cinq niveaux géographiques précis : le niveau régional (NUTS 2) est privilégié. La carte très hétérogène des collectivités locales en Europe est ainsi rationalisée. Il en résulte une plus grande prise en compte des problèmes régionaux en tant que tels mais aussi une promotion de la Région comme niveau pertinent des politiques publiques nationales et européennes ${ }^{10}$. La définition des « régions éligibles » est une opération politique très sensible, à la charnière des intérêts gouvernementaux et de la

\footnotetext{
${ }^{10}$ AMARAL, Carlos E. Pacheco. Regional Autonomy and International Relations. Paris : L'Harmattan, 2011. $324 \mathrm{p}$.
} 
politique de la Commission. Celle-ci distingue « région défavorisée » et « région prioritaire » à partir de critères comme le taux de chômage, le nombre et la qualité des infrastructures. S'y greffent des critères géographiques (les régions ultrapériphériques feront l'objet d'un «programme d'options spécifiques » en novembre 1988, mais aussi les zones arctiques). Si la réduction des disparités régionales reste la priorité, apparaît le souci de compenser les effets négatifs des politiques d'ouverture et de concurrence des économies régionales (par exemple la PAC aggrave la vulnérabilité des productions méditerranéennes). Et la perspective de l'élargissement aux trois pays du Sud ne fait que souligner l'importance de ce problème.

La présidence Delors va exploiter ces résultats. Le fonctionnement du FEDER est revu pour s'imposer comme l'instrument de développement pour les régions du Sud mais aussi les îles britanniques. Elle se prolonge avec la définition d'une politique de coopération transfrontalière autour de l'aménagement du territoire (et pas seulement les jumelages du Conseil de l'Europe): le FEDER prend le pilotage des projets comme SAARLORLUX (Sarre, Lorraine, Luxembourg) ou l'aéroport Bâle-Mulhouse. Elle y est encouragée par la signature de la Convention de Schengen en juin 1985. Elle mène à bien la reconversion de la sidérurgie du bassin de Longwy (à cheval sur trois Etats) mais aussi la création du Collège européen de technologie. C'est l'élargissement de la Communauté à la Grèce, l'Espagne et le Portugal qui va donner une nouvelle dimension à cette politique.

\subsubsection{Les Programmes Intégrés Méditerranéens (PIM)}

C'est une opération définie comme «pilote» par J. Delors dans la perspective d'une importante réforme des Fonds structurels ${ }^{11}$. Elle est portée par un contexte politique bien particulier, la chute des dictatures dans les trois pays : en avril 1974 au Portugal, en juillet en Grèce, suivie par la mort de Franco à la fin de 1975. Dans les démarches d'adhésion, la question agricole (vins, fruits et légumes) est essentielle tant pour la France que l'Italie et leurs régions méridionales (on parle à leur égard d'un « Mezzogiorno communautaire ») ; les politiques d'aides à ces régions apparaissent jusqu'ici comme inefficaces et onéreuses ce qui devrait s'aggraver avec l'accentuation de la concurrence suite à l'arrivée de l'Espagne notamment.

\footnotetext{
${ }^{11}$ DELORS, Jacques - Discours devant le Parlement européen (18 février 1987), Bulletin des Communautés européennes. 1987, $\mathrm{n}^{\circ}$ suppl. 1/1987. Luxembourg: Office des publications officielles des Communautés européennes.
} 
En mars 1983 la Commission a défini une proposition de PIM très ambitieuse : 6,6 Milliards (équivalents Euros) sur six ans. Jacques Delors la reprend dés son arrivée en la réduisant et la concentrant sur trois pays seulement : la Grèce en totalité, l'Italie (sauf Rome, Naples et Palerme), la France (cinq régions du Sud, sauf Bordeaux, Toulouse et Marseille) ${ }^{12}$. La stratégie est commune à ces territoires. La gestion est assurée directement par la Commission qui coordonne des actions interdépendantes impliquant les «autorités régionales ». C'est la Commission qui a la maîtrise de l'évaluation, du contrôle et de l'analyse des résultats.

Vingt-neuf PIM seront ainsi présentés par les trois Etats concernés. Ils seront la démonstration de la nécessité d'une évolution des fonds structurels vers une politique régionale pluriannuelle spécifiquement européenne.

La Commission Delors va s'appuyer sur ces résultats pour fonder la politique dite de cohésion qui va inspirer la réforme des Fonds structurels et leur quasi-fusion. Un Commissaire et une nouvelle Direction (la DGXXII) vont assurer la coordination et la gestion des trois Fonds (qui sont doublés) ainsi que des PIM. Ce sera le levier stratégique du grand bond en avant de la CEE avec la relance de la construction européenne. L'achèvement du marché intérieur apparait comme impossible sauf à assumer des déséquilibres régionaux considérables. D'évidence le Traité de Rome ne suffit plus. La Commission Delors lie ainsi les mesures de libéralisation de l'Acte unique au «renforcement de la cohésion économique et sociale »; ce qui implique la réforme des Fonds structurels; ce qui passe par «une politique d'aménagement du territoire européen au-delà des frontières nationales » (Déclaration de la Commission, 1987). Le FEDER est désigné comme le «pilote du développement régional au niveau communautaire ».

Tout cela a conduit à la mise en place d'une grande politique horizontale sous l'égide de la Commission qui a donné une illustration (restée quasi unique) d'un équilibre possible entre libéralisme et solidarité. Elle a aussi rendu crédible la perspective d'une politique euroméditerranéenne qui devait d'ailleurs aboutir à la Conférence de Barcelone les 27 et 28 novembre $1995^{13}$. Elle a enfin permis d'évacuer les aspects intergouvernementaux des règlements antérieurs qui entravaient la politique régionale à sa naissance. C'est une dénationalisation capitale de celle-ci qui a été ainsi réussie. Les pays membres ont d'ailleurs démantelé dans la foulée leurs organismes nationaux d'intervention régionale (Cassa per il

\footnotetext{
${ }^{12}$ ALLIES, Paul - Les régions du sud et les programmes méditerranéens de l’Union européenne. Pôle Sud, $n^{\circ} 3$. Automne 1995. Pp 141-146

${ }^{13}$ BISTOLFI, Robert (Ed.) - Euro-méditerranée, une région à construire. S.1. : Publisud, 1995. 339p.
} 
Mezzogiorno en Italie, DATAR en France...). La création du Comité des Régions par le traité de Maastricht en 1991 a laissé espérer une dynamique institutionnelle régionale. Les Etats y ont rapidement mis un terme. La phase qui allait s'ouvrir pour la politique régionale allait être marquée par des élargissements imprévus, inaugurée par la chute du mur de Berlin. Mais elle allait connaître, dans un contexte défavorable, un nouvel essor qui devait en faire une dimension incontournable et irréversible de la construction de l'Europe.

\section{2 - La dynamique d'un régionalisme fonctionnel}

La période 1989-2009 est celle de la mise en place d'un nouveau système de programmation de la politique régionale qui était inscrite à l'ordre du jour de l'expansion de celle-ci dans les décennies antérieures. Mais elle correspondait aussi à une transformation radicale du système : celle de l'unification de l'Allemagne, d'un premier élargissement (à trois nouveaux membres en 1995) suivi d'un second beaucoup plus vaste (de 15 à 27 en 2004). Cette expansion était aussi celle d'une nouvelle vague de Traités (Maastricht, Amsterdam, Lisbonne). La politique régionale va faire gagner à l'Union une dimension sociale essentielle dans sa légitimation auprès des populations des Etats-membres.

2.1. - La politique régionale comme socle de la construction européenne

La dynamique engagée par la Commission va se poursuivre à travers deux périodes de programmation 1989-93 et 1994-99, démontrant la supériorité d'une certaine planification européenne sur l'annualité budgétaire des Etats ${ }^{14}$. Portée par la présidence Delors (au-delà même de 1995), cette nouvelle programmation régionale va s'avérer efficace dans le traitement d'un premier élargissement jusqu'en 1999 donc.

\subsubsection{La performance d'une programmation régionale}

A l'origine (1988) une réforme des Fonds structurels est engagée : elle tablait sur la stabilité du communautaire à douze Etats membres et sur l'objectif prioritaire du rattrapage des régions du Mezzogiorno communautaire. La DG XVI-Regio est donc renforcée. Ses interventions sont affinées par la création d'unités plus petites (NUTS 4) du niveau des «bassins d'emploi ». Soient soixante zones sélectionnées par la Commission (sur les 900

\footnotetext{
${ }^{14}$ DREVET, op.cit. p. 85 et s.
} 
proposées par les Etats membres). La politique régionale va s'éprouver dans la prise en compte des problèmes des «zones pauvres des pays riches ». $43,5 \%$ de la population totale de l'UE est concernée par l'un des trois objectifs territoriaux dont les Fonds sont doublés (pour les régions de l'objectif 1- développement des infrastructures- c'est même un triplement pour le Portugal et un quadruplement pour l'Irlande). Globalement la Grèce, le Portugal, l'Irlande, l'Espagne et l'Italie sont (dans cet ordre) les grands bénéficiaires de cette politique financée d'abord par l'Allemagne (à 48\%), puis la France, les Pays-Bas et le Royaume-Uni. La réforme privilégie une approche régionale qui implique fortement les collectivités locales. Par ailleurs elle réoriente les interventions vers l'investissement productif (moins vers les infrastructures, que préfèrent pourtant les Etats), les actions de formation de la main d'œuvre liées aux opérations de reconversion. La Commission centralise comme jamais le suivi des programmes d'autant plus qu'elle n'a pas de contact direct avec le terrain ${ }^{15}$. Mais elle parvient ainsi à rationaliser la gestion : la totalité des budgets sont utilisés (malgré les carences de l'Italie); et surtout le niveau régional devient plus homogène en dépit de très grandes différences constitutionnelles (en Belgique la régionalisation est proche du séparatisme alors que la Portugal n'a pas encore alors de régions de plein exercice).

Ce résultat va connaître une mutation avec la chute du mur de Berlin ${ }^{16}$. Dés la fin de 1989 l'Allemagne (jusque-là hostile à la politique régionale européenne) sollicite celle-ci pour développer la coopération transfrontalière entre l'Est et l'Ouest. Les cinq nouveaux Länder de l'ex-RDA et Berlin sont immédiatement intégrés de plein droit dans l'Union sans période de transition. Ils représentent une population dont le niveau de vie est à 35\% de la moyenne UE. Un Tiers à peine des entreprises sont jugées encore compétitives. Les six entités font donc parties des régions les plus défavorisées et Bonn obtient 3 Milliards d'Euros forfaitaires dont la moitié pour le FEDER. Ils sont abondés par les Länder de l'Ouest qui consacrent 4,5\% de leur PIB à ce qui va devenir la plus grande politique régionale de l'histoire européenne. Cette expansion quantitative va avec une nouvelle réorientation des interventions vers l'amélioration de l'environnement. L'Allemagne devient un consommateur régulier des fonds régionaux et le deuxième bénéficiaire après l'Espagne.

\footnotetext{
15 DREVET, op.cit. p 96 et s.

${ }^{16}$ LARAT, Fabrice - Histoire politique de l'intégration européenne. Paris : La Documentation Française, 2003. $128 \mathrm{p}$.
} 
2.1.1.1. L'affirmation de la Commission dans la politique régionale

La Commission a de nouvelles marges de manœuvre et elle en profite pour piloter douze nouvelles initiatives ${ }^{17}$. Ainsi INTERREG participe dés 1990 d'une approche territoriale qui se manifeste principalement sur les régions ultrapériphériques, la coopération transfrontalière $(507.000 \mathrm{~km} 2$ et 53 Millions d'habitants), et la coopération interrégionale (850 collectivités locales) justifiée par la suppression des frontières internes de l'Union dans le cadre de l'achèvement du marché unique. Les Etats s'efforcent de contenir cette offensive en maintenant la politique régionale dans le cadre intergouvernemental puisque les traités ne prévoient pas de compétence communautaire dans le domaine de l'Aménagement du Territoire. L'issue sera dans un objectif défini en 1991 par la Commission (Europe 2000) et qui devient à la fin de 1993 un Schéma de Développement de l'Espace Communautaire (SDEC) ratifié par les Etats. Le FEDER va pouvoir traiter des fleuves (inondations ou sécheresses), des bassins maritimes (sécurité de la circulation, gestion des ports) de la lutte contre la pollution. Les pays du Nord (Allemagne, Danemark ...) ouvrent la voie avec un programme sur la Baltique qui servira de modèle pour l'Arc Atlantique. Cette régionalisation thématique crée 500.000 nouveaux emplois dans les pays les plus pauvres dont la croissance est abondée de $0,5 \%$ dans cette période. Les effets politiques sont très importants tant cette politique rencontre un consensus général : la Flandre belge qui rechigne déjà à financer la Wallonie paye sans récriminer pour les pays de la cohésion. Les aides nationales des Etats à leurs territoires s'orientent désormais vers les zones éligibles au FEDER.

La nouvelle programmation (1994-99) va bénéficier de ces résultats. Le Traité de Maastricht (adopté en décembre 1991 mais qui ne s'applique qu'à partir de novembre 1993) établit un rapport entre le marché, la monnaie unique et «la cohésion économique et sociale » qui devient un objectif en tant que tel de l'UE. Cela veut dire (art. 2) : « un progrès économique et social», «un niveau d'emploi élevé », «un développement équilibré et durable », « un espace sans frontières intérieures ». Un Fond de Cohésion est donc crée pour financer les projets dans le domaine de l'environnement et des réseaux trans-européens de transports. Un Comité des Régions doit suivre ces politiques. La Commission devra remettre tous les trois ans un « rapport de cohésion » assorti de propositions.

\footnotetext{
${ }^{17}$ SMITH, Andy- La Commission européenne en politique(s). Pôle Sud, n¹5. Novembre 2001. 156p.
} 
L'adhésion de l'Autriche, de la Finlande et de la Suède a lieu en $1995^{18}$. Ce sont trois pays qui ont déjà beaucoup développé des politiques originales en faveur de leurs régions arctiques et qui demandent qu'on les reconnaisse. Ce sera chose faite dans le Traité d'adhésion (création d'un Objectif $\mathrm{n}^{\circ}$ ). Les cinq autres pays bénéficiaires (Espagne, Grèce, Portugal, Irlande, Italie) demeurent gagnants : ils contribuent à $23 \%$ du budget, mais reçoivent $66 \%$ des Fonds structurels et de cohésion. Quant à l'Allemagne, elle reçoit désormais presque autant que la Grèce. Cet essor est dû à l'augmentation très forte des investissements du secteur privé dans ces programmes (27\% de leur montant total). Bref la démonstration est faite de l'efficacité de la politique régionale dans la construction européenne.

Le Fond de Cohésion crée par le Traité de Maastricht (art 129) vaut d'abord pour les quatre pays les plus pauvres (Grèce, Portugal, Espagne, Irlande) et pour l'environnement et les réseaux transeuropéens de transports. Mais ils n'ont pas le choix de leurs secteurs d'intervention. C'est la Commission et le FEDER qui le font à leur place. Le résultat en 1999 sera là aussi très convaincant : le Fonds s'est avéré être un puissant stimulant à la croissance des dépenses d'environnement et de transports dans ces quatre pays qui ont pu rattraper leur retard (187.000 emplois directs crées et 56.100 indirects).

Le Traité d'Amsterdam reprend donc la démarche pour les régions ultrapériphériques (sept régions concernées) et pour la coopération transfrontalière (gestion des eaux et aménagement du territoire $)^{19}$. 59 programmes ont concerné plus de 10.000 projets de désenclavement et d'amélioration du tissu économique. La réduction des handicaps des régions transfrontalières a été spectaculaire dans les domaines de la scolarisation, la santé, la formation. La frontière n'était plus un écran mais un foyer d'attractivité.

On comprendra aisément que la projection «Europe 2000 » soit inspirée par cet eurorégionalisme. La Commission y proposait quatorze programmes opérationnels et une répartition du territoire communautaire en sept groupements en fonction de périmètres de coopération significatifs et forgés par ces «bonnes pratiques ». Le résultat ne sera pas mince : 4100 kilomètres d'autoroutes et 32.000 de routes, maîtrise des eaux... Les régions de l'Objectif 1 sont passées de 64\% à 69\% de la moyenne PIB-UE par habitant. La politique régionale communautaire s'est imposée comme plus efficace et moins onéreuse que celle menée par les seuls Etats (surtout en Italie et en Allemagne), capable en outre de favoriser l'investissement public-privé et la mobilisation des collectivités locales. Et cela a concerné

\footnotetext{
${ }^{18}$ OLIVI, Bino, GIACONE Alessandro - L’Europe difficile. La construction européenne. Paris : Gallimard, 2007. 546p.

${ }^{19}$ STIRK, Peter. WEIGALL, David - The origins and Development of European integration. A Reader and Commentary. London : Pinter, 1999. 233p.
} 
plus de la moitié du territoire et de la population communautaire. Mais elle a buté sur le chômage et la fragilisation des zones rurales. La donne aller changer avec le grand élargissement de la période 2000-2005.

2.2. .- La victoire du grand marché sur la cohésion interrégionale

Les douze pays entrants de l'Europe centrale et de l'Est présentent un ensemble de problèmes inédits qui vont être aggravés par l'absence de méthode de l'élargissement. L'approfondissement de l'intégration politique ayant été sacrifié, c'est dans la précipitation que va s'opérer le passage de l'UE de 15 à 27 membres, en partie du fait de crises majeures comme la guerre dans l'ex-Yougoslavie.

\subsubsection{Les effets pervers de l'élargissement :}

Concrètement, il s'agit de réhabiliter un million de kilomètres carrés marqués par une gestion d'espaces sinistrés, depuis l'insécurité routière jusqu'à l'insécurité nucléaire. Si la main d'œuvre est éduquée, elle n'est pas forcément qualifiée. Le PIB est beaucoup plus bas que ceux des pays méditerranéens : au moment de leur adhésion : moins de 50\% au lieu de $70 \%$. L'écart entre les régions est beaucoup plus grand si bien que les fonds structurels sont déterminants pour assurer la viabilité de l'élargissement. Or ces pays n'ont aucune politique régionale : pas d'administration, pas de niveau NUTS 2 (sauf la Pologne et la Roumanie qui possèdent des régions, mais sans compétences réelles tous les autres pays n'ont que des NUTS 3 -niveau départemental- ou pas d'échelon intermédiaire du tout vu leur taille -Pays Baltes, Slovaquie...). Alors que la transition économique est connue pour être un facteur d'aggravation des disparités, les gouvernements de ces pays privilégient l'entrée dans l'économie de marché avec des recettes libérales qui les détournent d'une prise en compte de la dimension régionale et de la cohésion sociale qui l'accompagne.

Tout cela fait que la Commission place son action dans le cadre du programme PHARE défini en 1988 pour la Pologne et la Hongrie. Il était fait pour faciliter le retour de ces pays à l'économie de marché sans engagements pluriannuels ni programmes. Un début de coopération transfrontalière était engagé dans le cadre d'INTERREG mais, là non plus il n'avait pas donné lieu à de vrais projets structurants. C'est dire que la Commission elle-même se trouvait démunie, ses Directions Générales n'étant même pas associées aux négociations d'intégration pays par pays. C'est que les quinze Etats de l'UE redoutaient le coût excessif 
d'un élargissement qui allait faire passer la population des régions concernées par l'Objectif 1 de 25 à $42 \%$ de la population totale de l'Union. Et ils ne veulent pas payer à la fois pour l'Est et le Sud où le processus d'intégration n'est pas achevé. Qui plus est, le contexte est plutôt devenu celui de la rigueur budgétaire dans le cadre de la réalisation de l'union monétaire. Il apparaît clair que l'Europe centrale ne bénéficiera pas du même effort en volume que l'Europe du Sud ni, non plus des politiques qui y ont réussi jusque-là.

Il y a donc un double registre qui s'ouvre: d'une part l'achèvement de l'UE à quinze ; d'autre part la réalisation à court terme de l'élargissement aux dix nouveaux Etats ${ }^{20}$.

Pour l'UE à 15, on fige la carte du déclin industriel : au lieu d'être des zones temporaires de courte durée, elles sont fixées pour sept ans comme si le déclin industriel devenait un élément permanent d'une politique structurelle dont on ne croît plus qu'elle peut le résorber. Les Fonds structurels sont maintenus en l'état au prix d'une baisse de $2 \%$ de leur dotation. Les quatre pays de la cohésion (ainsi que le Fond de cohésion) maintiennent leurs parts (l'Espagne et la Grèce l'améliorant même quelque peu). Ainsi le FEDER confirme ses qualités et compétences dans la gestion des crédits d'engagement. Le Fond de cohésion a tiré son épingle du jeu. Les Fonds structurels s'avèrent efficaces pour soutenir l'investissement public : dans les pays de la cohésion, il a augmenté de 3,5\% du PIB alors qu'il a reculé de 2,9\% dans le reste de l'UE à 15. Le rôle de la Commission s'est renforcé en amont dans la préparation des règlements; il a diminué dans la phase de mise en œuvre.

Pour l'élargissement aux dix nouveaux pays (la négociation s'est engagée en 2000 pour six d'entre eux) la date de leur adhésion devient un objectif politique en soi, même si elle est irréaliste et si les conditions de préparation ne sont pas réunies. Les conditions de préadhésion telles que gérées par Bruxelles sont perçues par les administrations de ces pays comme abusives. Ces administrations sont instables, marquées par un turn-over inhabituel (vu l'attraction du secteur privé et l'impact de l'instabilité ministérielle). L'assistance technique des services de la Commission, même massive comme en Roumanie, n'est pas mise à profit. La politique régionale est donc passée par profits et pertes. La mise en œuvre des Fonds structurels est problématique (inférieure à 30\% des engagements du FEDER). La Commission la délègue aux gouvernements centraux qui découragent les quelques mouvements de décentralisation possibles et nécessaires. Les inégalités entre pays sont importantes, la Pologne, la Hongrie et la République Tchèque étant les plus gros bénéficiaires. Un document de la Banque mondiale de 2006 souligne le contraste entre les mêmes politiques au Sud et à

\footnotetext{
${ }^{20}$ BOILLOT, Jean-Joseph - L'Union européenne élargie. Un défi économique pour tous. La Documentation française. Paris : S.e., 2003. 149p.
} 
l'Est où les incitations à la modernisation se sont détachées des politiques régionales de cohésion comme en témoigne la faible consommation des Fonds. Et elle souligne l'importance du professionnalisme et de la qualité des administrations publiques dans ces processus.

\subsubsection{La résistance du régionalisme européen}

Finalement c'est le FEDER qui va être, même par défaut, la structure essentielle de cet élargissement Il assure la consolidation des acquis tant pour les régions ultrapériphériques que pour la coopération transfrontalière. INTERREG a été profondément réorganisé et étendu avec des spécifications pour les régions maritimes ou insulaires, les Balkans... Elle a confirmé son rôle de catalyseur pour les politiques territoriales, 23 régions étant concernées.

Néanmoins elle n'a pas réglé le problème majeur de l'écart entre l'Est et l'Ouest (entre l'UE à 15 et l'UE des 12 nouveaux): le PIB par habitant est de $115 \%$ à l'Ouest par rapport à la moyenne UE à 27 et 53\% à l'Est (37\% pour les pays les plus à l'Est). C'est plus que de part et d'autre de la frontière entre les Etats-Unis et le Mexique. En 2003 la «politique européenne de voisinage » a été définie pour remédier à cela par la systématisation de la coopération transfrontalière et l'encouragement des Eurorégions qui sont la principale réussite en matière de coopération internationale concentrée sur l'environnement et la construction de réseaux transeuropéens ${ }^{21}$. La Commission a divisé l'espace européen en quatre grande zones bénéficiant chacune d'un programme. Cela devait permettre de gérer au mieux l'agenda de Lisbonne (l'Europe de la connaissance, adopté en mars 2000). Mais les dépenses afférentes étaient faiblement spatialisées ou concentrées dans les grandes villes, hors zones éligibles. L'inapplication de ce projet a fait qu'il n'a eu aucun effet dans l'Europe élargie.

Au terme de cet examen, on observera l'avancée que constitue la mention de la « cohésion économique et sociale » ainsi que de la «cohésion territoriale » définis de manière extensive dans les Traités de Nice puis de Lisbonne. INTERREG est un donc devenu un axe permanent et à part entière de la construction de l'UE.

Mais cela est allé avec une remise en cause du principe de l'aide aux régions défavorisées et d'une politique régionale en tant que telle. Le rapport Sapir (un économiste belge ultralibéral) commandé par la Commission Prodi en 2002 a consisté à une remise en question complète de la politique structurelle et sa régionalisation. Il n'a fait que théoriser ce qui s'est

\footnotetext{
${ }^{21}$ BOLGHERINI, Sergio - Come le regioni diventano europee. Bologna : Il Mulino. 2006. 342p.
} 
passé depuis : une renationalisation de cette politique qui correspond bien au retour d'une Europe inter-gouvernementale où la puissance a abdiqué devant le marché. Et la stratégie Europe 2020 n'a pas, jusqu'ici infléchit cette tendance.

L'avenir de la politique régionale est donc tributaire de quatre facteurs :

- Politique : l'accord des Etats sur le principe même d'une régionalisation de l'UE et l'acceptation d'une sorte de « discrimination territoriale positive ».

- Budgétaire : le financement suffisant des engagements structurels pour que la Commission puisse procéder à une répartition significative

- Technique : l'opérationnalité des organes « régionaux » de la Commission en dépit de la «gouvernance multi-niveaux » et la complexité des 27 membres de l'UE

- Économique : la réduction des disparités ne suffit plus; il faut pouvoir dégager une valeur ajoutée communautaire.

En d'autres termes, de la même manière qu'il y a un coût de la non-Europe, il y a un coût de la non-solidarité chiffré à $0,2 \%$ du PIB (alors qu'il devrait atteindre $0,6 \%$ pour garantir sans inconvénient des transferts efficaces). Il s'agit donc de savoir si les gouvernements sauront s'assigner ces objectifs ${ }^{22}$. S'ils ne le font pas, sur un terrain proche des préoccupations de leurs populations, on peut craindre que les opinions publiques se détournent de l'idée fondatrice de l'Europe de l'après-guerre. Les guerres d'ailleurs y ont refait leur apparition à ses frontières alors même que l'UE semble avoir renoncé à toute politique de défense commune si ce n'est d'approfondissement institutionnel ${ }^{23}$. Sur ce dernier plan, l'issue démocratique serait une solution qui supposerait un agencement autrement plus construit que ce que contient le traité de Lisbonne. La manière dont l'Union s'est engagée à marche forcée dans des politiques d'austérité a atteint ici et là le cœur des sociétés qui se les sont vues appliquées. La politique régionale est devenue une variable d'ajustement et la politique de cohésion un moyen pour orienter autant que possible l'investissement vers la «croissance verte » et non plus pour soutenir le développement régional. Finalement, la politique régionale est un acteur parmi d'autres de renationalisation au sein d'une Union gouvernée par quelques «grands Etats » et leur stratégie de compétitivité telle que définie dans «Europe 2020 ». Il semble donc bien que le cycle vertueux de la régionalisation ait épuisé ses effets dans ce que l'UE est devenue. Mais son histoire reste riche d'enseignements pour les jours heureux de la relance du projet européen.

\footnotetext{
${ }^{22}$ MAGNETTE, Paul. Le régime politique de l'Union Européenne. Paris : Presses de Sciences Po, 2003. 286p.

${ }^{23}$ DEHOUSSE, Renaud. La fin de l'Europe. Paris : Flammarion., 2005. 191p.
} 